\title{
The Geometry of the Circle of Willis Anatomical Variants as a Potential Cerebrovascular Risk Factor
}

\author{
Raluca PASCALAU ${ }^{1 *}$, Vlad Adrian PADUREAN ${ }^{1 *}$, Dana BARTOS ${ }^{2,3^{*}}$, Adrian BARTOS ${ }^{3^{*}}$, Bianca Aurora SZABO ${ }^{2,4^{*}}$ \\ 1"Iuliu Hatieganu" University of Medicine and Pharmacy, Faculty of Medicine, Cluj-Napoca, Romania \\ 2"Iuliu Hatieganu" University of Medicine and Pharmacy, Department of Anatomy and Embryology, Cluj-Napoca, Romania \\ ${ }^{3}$ Regional Institute of Gastroenterology and Hepatology, Department of Surgery, Cluj-Napoca, Romania \\ ${ }^{4}$ Emergency County Hospital Cluj, Department of Ophthalmology, Cluj-Napoca, Romania
}

"Raluca PASCALAU, Vlad Adrian PADUREAN, Dana BARTOS, Adrian BARTOS and Bianca Aurora SZABO have contributed equally to conceiving, writing and reviewing the manuscript so that they should all be considered as main authors.

Corresponding author: Raluca PASCALAU raluca.pascalau@yahoo.ro

\section{ABSTRACT}

AIM: To correlate the anatomical variants of the circle of Willis with their effects on the hemodynamic and geometrical parameters responsible for the pathogenesis of neurological diseases.

MATERIAL and METHODS: The circle of Willis and the proximal segments of the main arteries were dissected and measured on ten formalin-fixed human brains. The anatomical variants were systematized using descriptive statistics. The mathematical models for brain perfusion and wall shear stress were developed by optimally approximating resistance to flow, vascular conductance, and branching.

RESULTS: Eighty percent of the brains presented asymmetries, especially in the posterior communicating (70\%) and anterior cerebral (40\%) arteries. The posterior circulation had more variations (65.21\%). Nine hypoplastic vessels were found in 7 brains. Atypical origins were observed in eight specimens. According to the mathematical models, which integrated each anatomical change in the global circle of Willis anatomy, the circle of Willis' geometry could represent a risk factor for intracranial aneurysms and atherosclerosis, mostly when hypoplastic arteries are present, due to high resistance to flow and imbalanced bifurcation geometry. Accessory vessels are less associated with cerebrovascular risk.

CONCLUSION: We described anatomical variants of both the anterior and posterior circulations and their specific effects on the hemodynamic balance of cerebral blood flow.

KEYWORDS: Anatomical variant, Circle of Willis, Perfusion, Shear stress

\section{INTRODUCTION}

$\mathrm{T}$ The circle of Willis is the anastomotic system that connects the four sources of brain vascular supply: The two internal carotid arteries (ICA) and the two vertebral arteries (VA) (18). It is formed by the basilar artery (BA), the first segments ( $\mathrm{P} 1)$ of the posterior cerebral arteries (PCA), the posterior communicating arteries (PCoA), the first segments (A1) of the anterior cerebral arteries (ACA) and the anterior communicating artery (ACoA) (22). The major branches arising directly from the circle of Willis are the anterior inferior cerebellar arteries, the superior cerebellar arteries ( $\mathrm{SCbA})$, the second segments of the posterior cerebral arteries, the middle cerebral arteries (MCA), and the second segments of the anterior cerebral arteries (Figure 1) (33).

The circle of Willis is a highly variable anatomical complex. The classical anatomical variant is symmetrical with a complete contour $(11,21)$, while $50 \%$ of the healthy brains (1) and $80 \%$ of the dysfunctional brains present other variants (12).
Raluca PASCALAU (D) : 0000-0002-7059-6896 Vlad Adrian PADUREAN (D): 0000-0002-9593-9859
Dana BARTOS (D) : 0000-0002-7523-4747

Adrian BARTOS (1) : 0000-0003-3177-9232
Bianca Aurora SZABO (D) : 0000-0002-2605-8513 


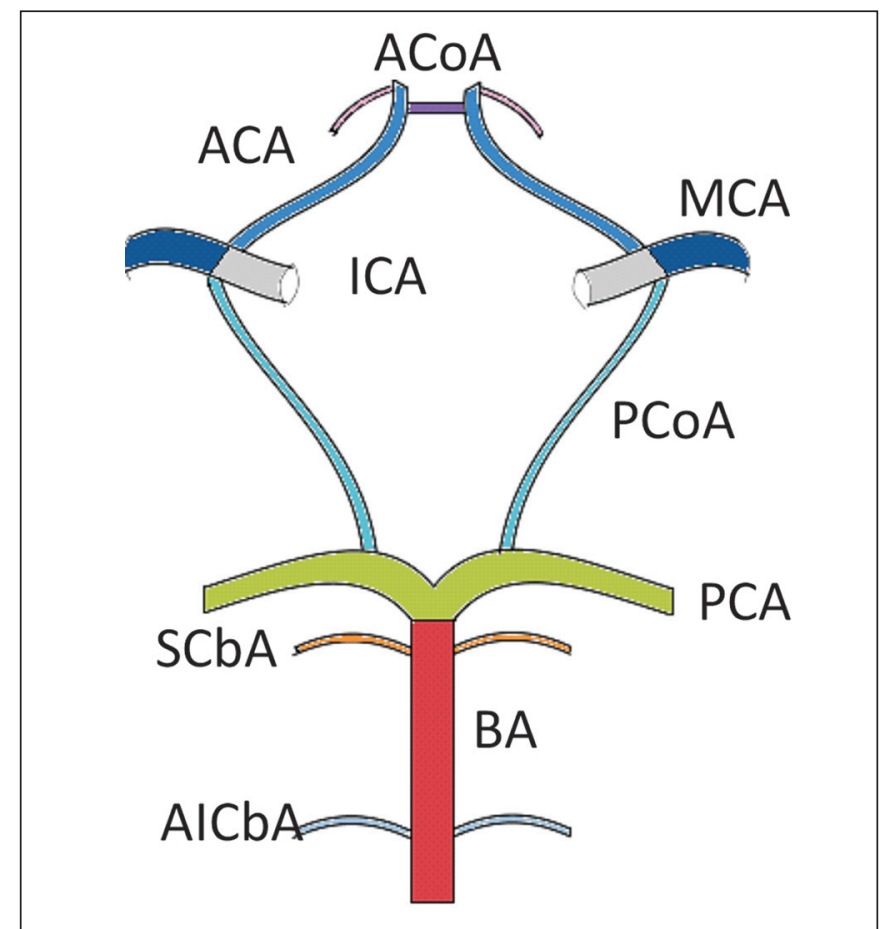

Figure 1: Circle of Willis. ACA: anterior cerebral artery; ACoA: anterior communicating artery; AICbA: anterior inferior cerebellar artery; BA: basilar artery; ICA: internal carotid artery; MCA: middle cerebral artery; PCA: posterior cerebral artery; PCoA: posterior communicating artery; SCbA: superior cerebellar artery.

Although the anatomical variants do not directly impair brain perfusion, they may increase the risk of neurological diseases by two main mechanisms: they may influence collateral perfusion $(5,8,37)$, leading to vascular and neurological surgery complications $(1,12,19,27,30,38)$, cerebral infarcts $(3,17)$, migraine $(9,10)$, or even psychiatric diseases $(14,23)$, or the imbalanced branching geometry present in some variants can predispose to wall shear stress $(2,5,29)$, associated with the formation and rupture of intracranial aneurysms $(6,13,26,29)$, and with atherosclerosis $(7,29)$. How each particular variant influences these processes is not fully understood. Thus, this study aimed to investigate the correlations between the characteristics of the arteries and branching points of some anatomical variants and the geometric features known to increase the cerebrovascular risk.

\section{MATERIAL and METHODS}

This study followed the provisions of the Declaration of Helsinki, 1995 (as revised in Edinburgh, 2000). Ten formalinfixed human brains that were held for teaching purposes in the anatomy collection of the "Iuliu Hatieganu" University of Medicine and Pharmacy, Cluj-Napoca, Romania, were used. The research protocol was approved by the Ethics Committee of the "Iuliu Hațieganu" University of Medicine and Pharmacy Cluj-Napoca (registration number 197/2016). Since the brains have been kept separated from the bodies for many years, it was not possible to ascertain the causes of death. However, cerebrovascular causes could be excluded as the brains showed no pathological processes. The study included a total of 120 arterial segments and 90 branching points. The circle of Willis and the proximal segments of the main arteries were dissected, and their diameters and lengths were measured using a Vernier caliper. The bases of the brains were photographed with a Nikon COOLPIX L120 camera from three standard angles: $20 \mathrm{~cm}$ parallel to the plane containing the anterior face of the pons and the two temporal poles, $15 \mathrm{~cm}$ parallel to the orbital gyri, and $15 \mathrm{~cm}$ parallel to the anterior surface of the brainstem. Anatomical variants such as asymmetry, hypoplasia, atypical origin, fusion, absence, or duplication were recorded and tabulated using descriptive statistics.

To assess hemodynamics and wall shear stress, the resistance to flow $(\mathrm{R})$ was calculated using the Hagen-Poiseuille law (28):

$$
\mathrm{R}=8 \eta \mathrm{l} /(\pi \mathrm{r} 4)
$$

where $\eta$ is the blood viscosity, I is the length of the arterial segment, and $r$ is the radius of the vessel. The vascular conductance $(C)$ corresponds to the inverse of the resistance to flow (16):

$$
C=1 / R
$$

To determine the optimality of the branching points, a bifurcation geometry model was used (40), according to which the apex of the bifurcation divides the blood flow of the parent artery (represented by its diameter $\mathrm{d} 0$ ) into two streamlines (c1 and c2) (Figure 2). The relation between the ratio of the branch arteries' diameters ( $d 1$ and $\mathrm{d} 2$ ) and the position of the flow divider is determinant for the branching optimality $(20,36,40)$.

Thus, the segments $\mathrm{c} 1$ and $\mathrm{c} 2$ were measured and the observed position of the flow divider ( $\gamma 0$ ) was expressed as:

$$
\gamma \mathrm{O}=\mathrm{c} 2 / \mathrm{c} 1
$$

The optimal position of the flow divider $(\gamma t)$ was obtained using a theoretic curve for the optimal position of the flow divider to the minimum lumen surface wall shear stress as a function of the ratio of the branch diameters (d2/ d1) (36). To determine the relative perception of the branching optimality in all bifurcations, the percentage departure from optimality (B) was calculated through the following formula:

$$
B=[(\gamma O-\gamma t) / \gamma t]{ }^{*} 100
$$

Finally, the Microsoft Excel 2010 and the Xara Photo \& Graphic Designer were used to build models of brain perfusion and wall shear stress, which integrated the resistance to flow, vascular conductance, and percentage departure from optimality, for each arterial circle. The use of color scales and radar charts are presentation of the risk distribution within the circle of Willis.

\section{RESULTS}

\section{Anatomical Variants}

A high arterial variability was found on the basal view of the brains (Figure 3). Each brain presented at least one morpho- 


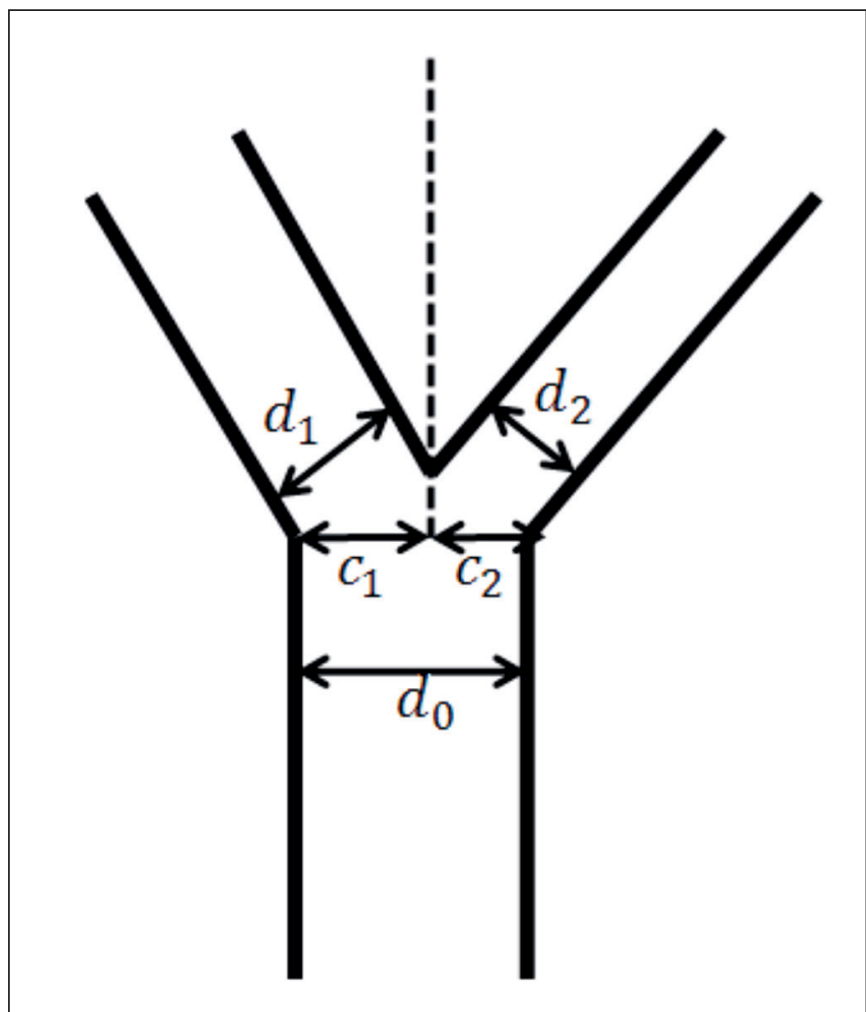

Figure 2: Bifurcation geometry model after Zamir (1982)(40). The apex of the bifurcation divides the blood flow of the parent artery (represented by its diameter $d_{0}$ ) in two streamlines $\left(c_{1}\right.$ and $c_{2}$ ). The diameters of the branches are $d_{1}$ and $d_{2}$. logical change. Moreover, $40 \%$ of the brains presented multiple anomalies. Anatomical variants occurred mainly in the posterior half of the circle, and the most frequent were asymmetry, hypoplasia, and atypical origins, occurring especially in the posterior communicating, anterior cerebral and superior cerebellar arteries (Figure 4). Accessory or absent vessels were also found, including the double superior cerebellar artery, the double or absent anterior inferior cerebellar artery, and the absent anterior communicating artery with fusion of the anterior cerebral arteries forming a single trunk, which divided into the two pericallosal arteries (Figure 5).

\section{Geometric Characteristics}

The diameter of the proximal and distal segments, as well as the length of each artery within the circle of Willis and the internal perimeter of the circle of Willis were measured and the mean values were calculated (Table I).

Graphical models illustrating the differences in the relative diameter of the arterial segments in each specimen were used to mark each segment according to its resistance to flow and its departure from branching optimality (Figure 6). This was done using a gradient of colors ranging from dark red to dark blue. At the branching points, the color code represents the value of the departure from branching optimality, whereas in the arterial segments the color represents the resistance to flow. The darkest red represents the highest resistance to flow and greatest departure from branching optimality, thus representing the areas that are most likely to become a risk in cerebrovascular disease. At the opposite pole, the darkest

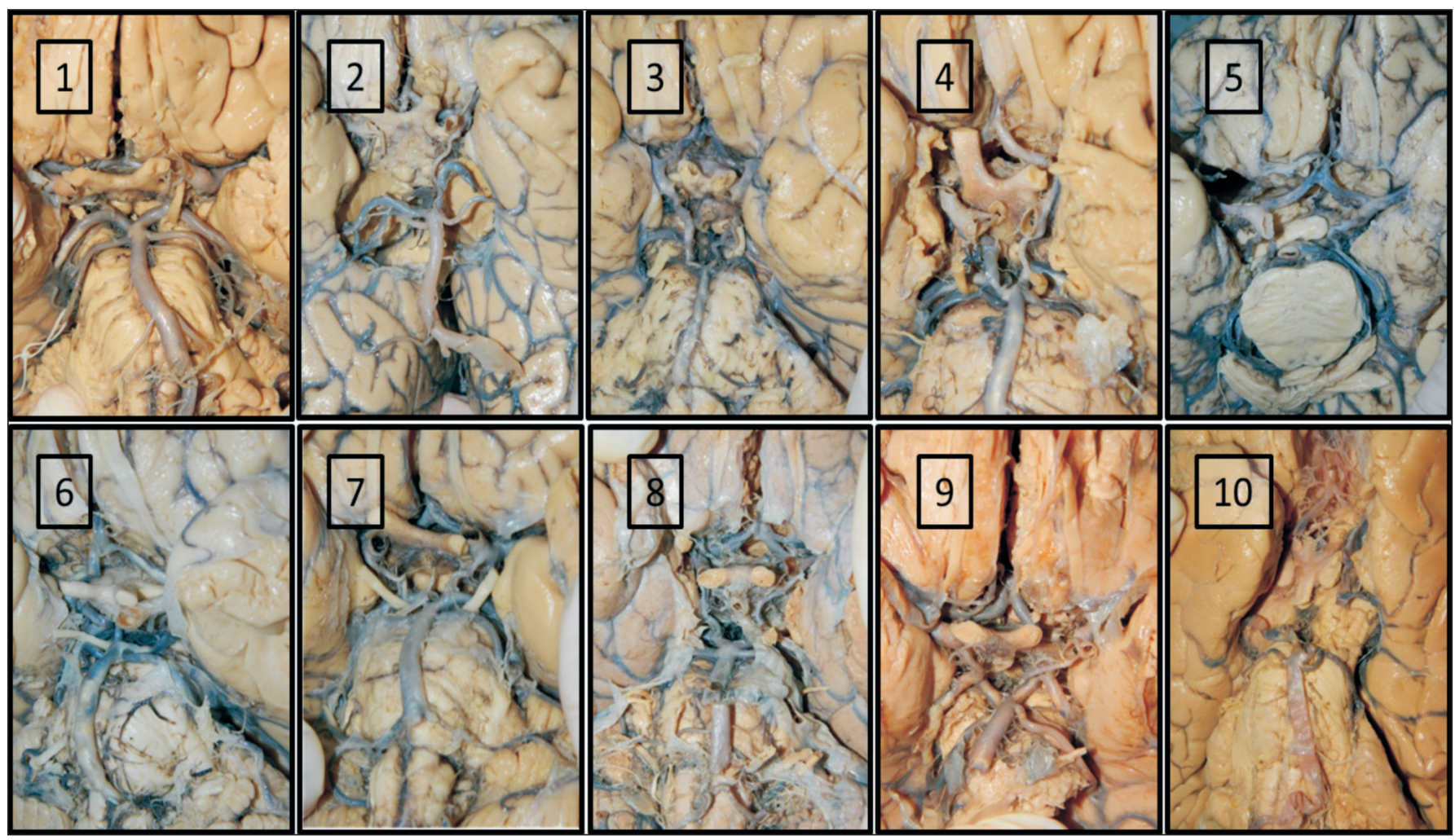

Figure 3: Basal surfaces of the ten formalin fixed human brains. 


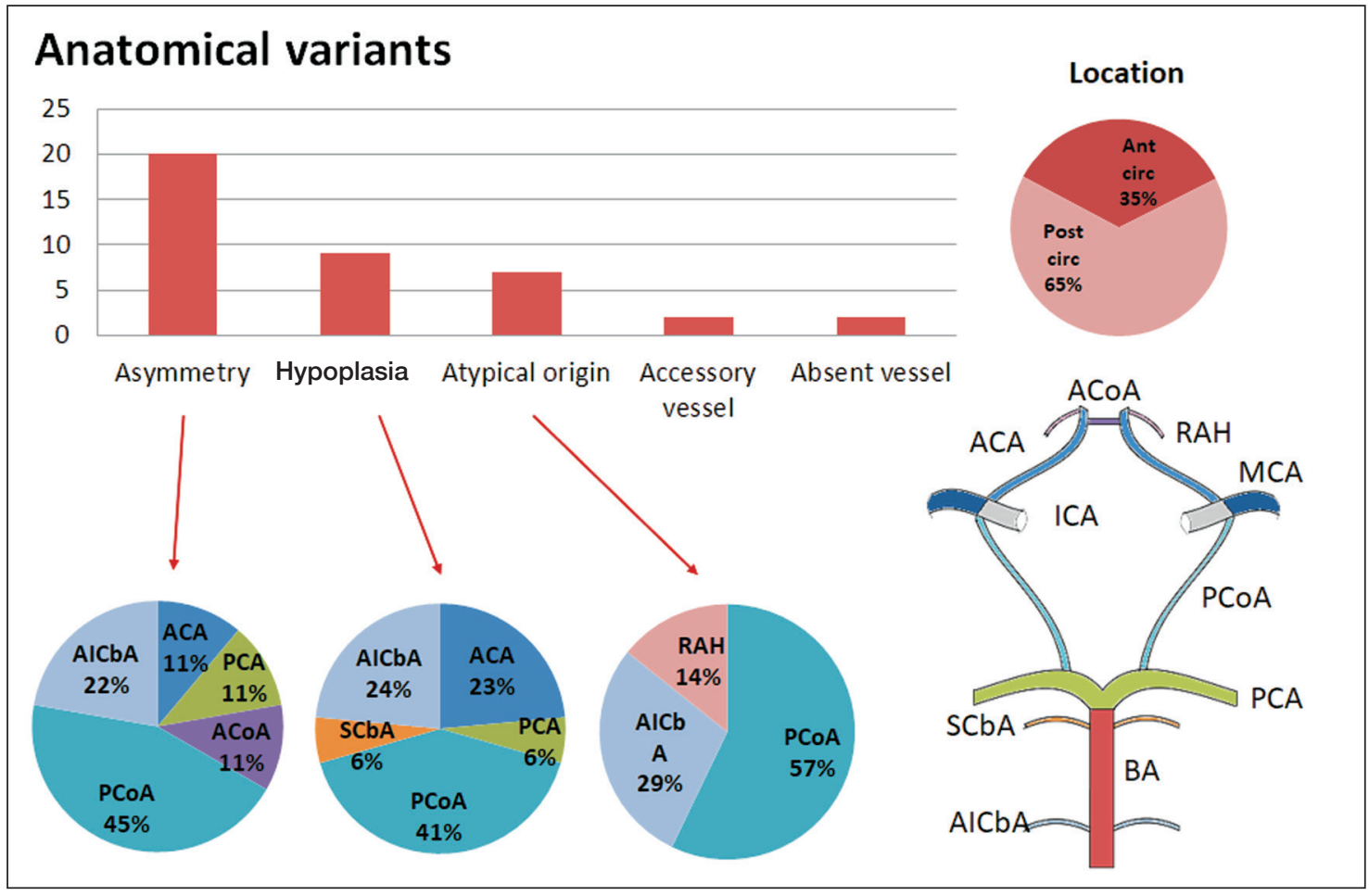

Figure 4: The distribution of the anatomical variants.

ACA: anterior cerebral artery;

ACoA: anterior communicating artery;

AICbA: anterior inferior cerebellar artery;

ICA: internal carotid artery;

MCA: middle

cerebral artery;

PCA: posterior

cerebral artery; PCoA: posterior communicating artery;

RAH: recurrent artery of Heubner; SCbA: superior cerebellar artery.

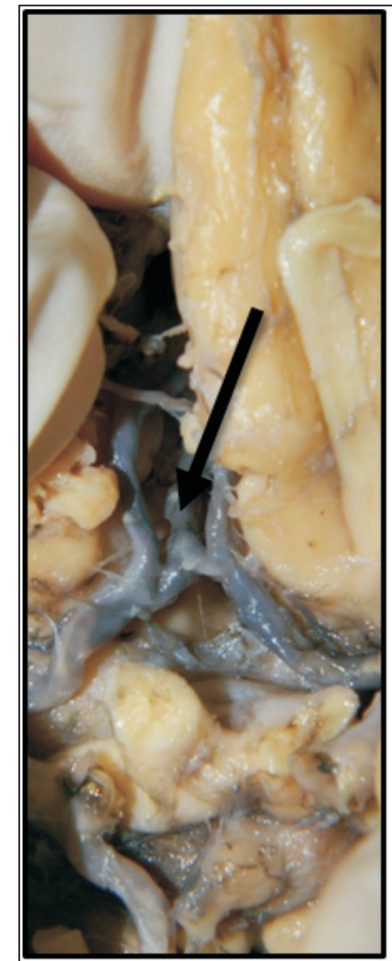

median callosal artery

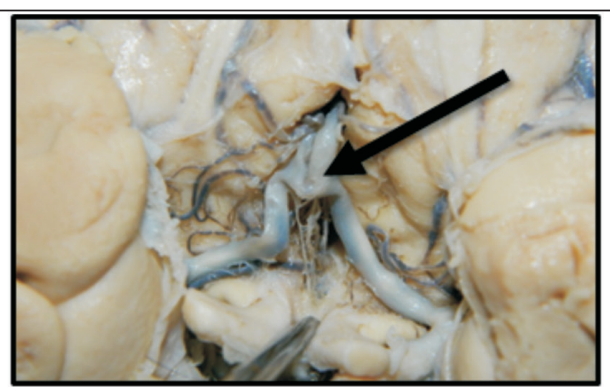

fusion of the anterior cerebral arteries

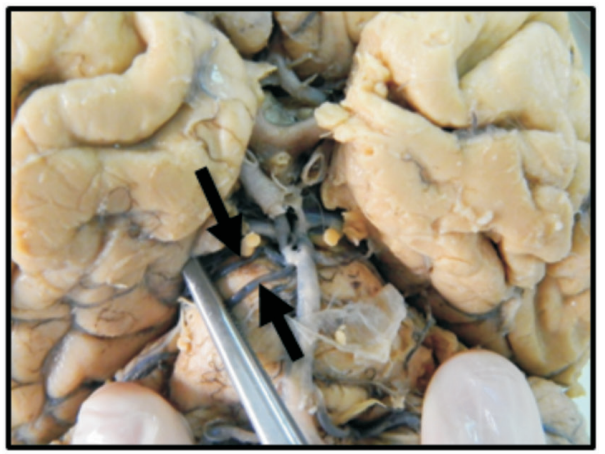

duplicated superior cerebellar artery

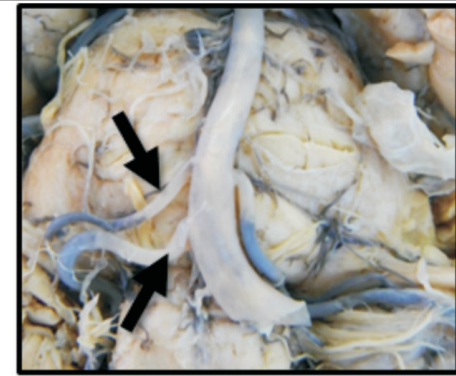

duplicated anterior inferior cerebellar artery

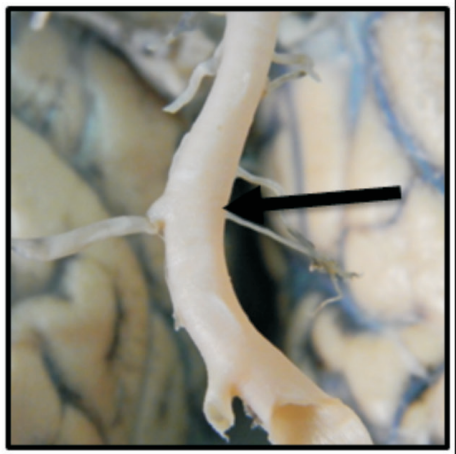

absent anterior inferior cerebellar artery
Figure 5: Examples of anatomical variants encountered among the brain specimens. Each variant is indicated with arrows. 
Table I: Mean Diameters and Lengths of the Arteries and Mean Perimeter of the Arterial Polygon

\begin{tabular}{lccc}
\hline Artery & $\begin{array}{c}\text { Mean proximal } \\
\text { diameter } \\
(\mathbf{m m})\end{array}$ & $\begin{array}{c}\text { Mean distal } \\
\text { diameter } \\
(\mathbf{m m})\end{array}$ & $\begin{array}{c}\text { Mean } \\
\text { length } \\
\mathbf{( m m})\end{array}$ \\
\hline ICA & - & 4.35 & - \\
\hline ACA & 2.7 & 2.52 & 14.63 \\
\hline ACoA & 2.18 & - & 2.31 \\
\hline MCA & 3.85 & - & - \\
\hline PCoA & 1.51 & 1.52 & 12.98 \\
\hline PCA & 2.59 & 3.9 & 5.89 \\
\hline BA & - & 4.29 & 26.48 \\
\hline $\begin{array}{l}\text { Mean perimeter } \\
\text { (mm) }\end{array}$ & & & 64.54 \\
\hline
\end{tabular}

blue stands for the areas least prone to become involved in pathological processes. In addition, the vascular conductance values were integrated into radar charts. The interpretation of the charts results from the analysis of the distance between the red line and the center of the chart-the higher the distance, the better the conductance, and vice-versa. The mean values for the conductance, resistance and branching optimality were integrated into a single model.

By comparing the different types of arteries in terms of the mean resistance to flow and the mean departure from branching optimality (Figure 7A, B), we observed different behaviors. First, hypoplastic arteries presented a higher resistance to flow and a greater departure from branching optimality than arteries within the circle of Willis. Second, the atypical origin of some vessels affected their branching optimality but not their conductance. Asymmetric arteries are resistant to blood flow to a lower extent than hypoplastic arteries and have imbalanced bifurcation geometry to a higher extent than hypoplastic arteries. The global resistance to blood flow throughout

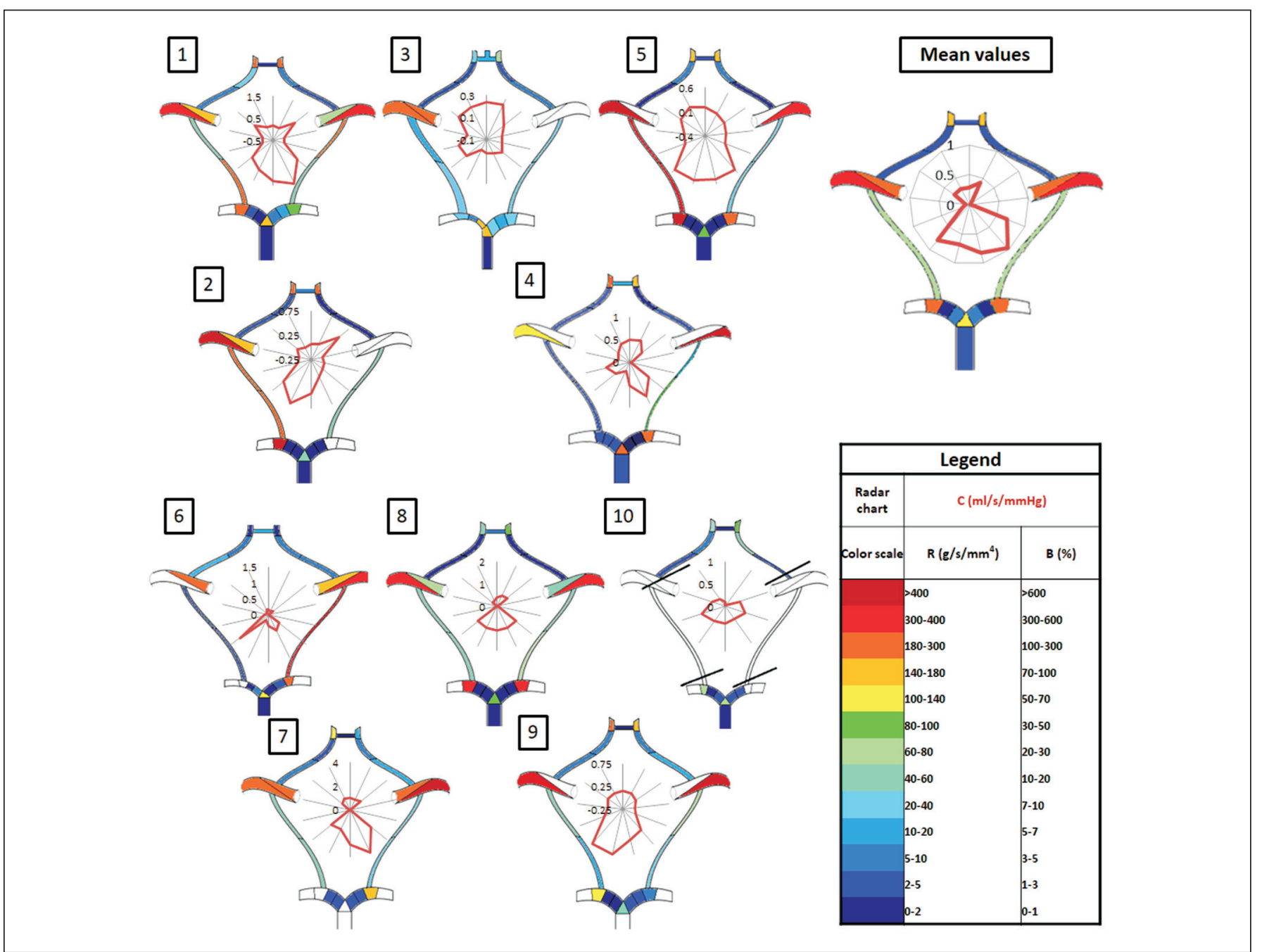

Figure 6: Graphical models. The color code corresponds to the departure from branching optimality to the branching points and to the resistance to flow in the arterial segments. Vascular conductance was represented with radar charts. C: conductance; R: resistance to flow; B: percentual departure from branching optimality. 
the circle of Willis enables for a good vascular conductance, except for the posterior communicating arteries, which have the lowest diameter among the other arteries and are often hypoplastic.

\section{DISCUSSION}

The anatomical variations of the circle of Willis found in this series of brain specimens are representative of those occurring in the general population. We found that variations were more frequent in the posterior circulation, the PCoA was the most variable artery, and the most common change was hypoplasia, followed by accessory vessels and arteries with atypical origin. Our results are in line with those of previous studies conducted on larger numbers of cadaveric specimens $(21,23,24,30,32)$, and in vivo studies using magnetic resonance (MR) $(19,22,39)$, or computed tomography (CT) angiography (41). In previous imaging-based studies, the frequency of absent vessels was higher than in our study and other previous cadaveric studies. On the other hand, the frequency of hypoplastic vessels was higher in our study and previous cadaveric studies than in imaging studies. The fact that hypoplastic vessels visualized and documented in cadaveric studies have no detectable blood flow may account for these differences since those vessels are usually classified as absent by imaging studies (41). In other words, those vessels are morphologically hypoplastic, but functionally absent. Most previous studies did not assess asymmetry. One exception was a study by Gunnal et al., who found that asymmetry was more frequent in the PCoA (38\%) followed by $A 1(31 \%)$ and P1 (22\%) (15), which is consistent with our findings.

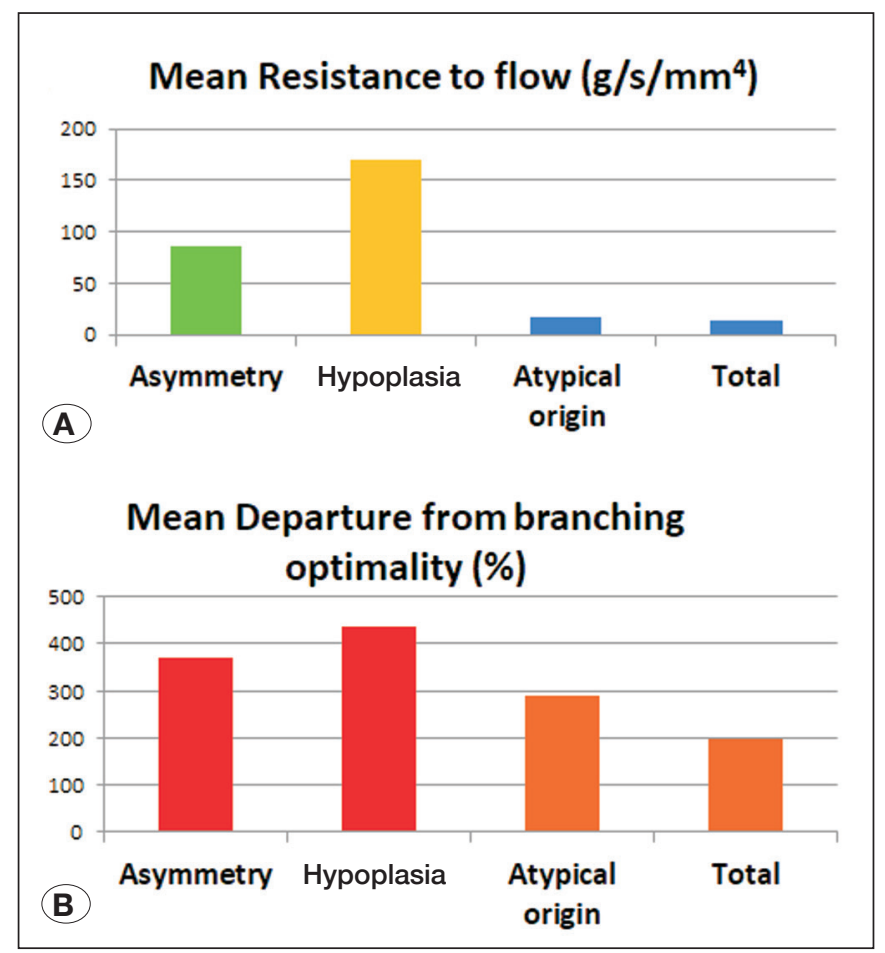

Figure 7: A) Mean resistance to flow. B) Mean departure from branching optimality.
The circle of Willis is meant to provide collateral perfusion when an artery is occluded (24). Among patients with stroke, those presenting the normal circle of Willis variant experience lower severity, recurrence rate and mortality, as well as a better recovery (22). Nevertheless, the different anatomical variants exert a crucial effect on the hemodynamic balance. Thus, we analyzed the geometry of the anatomical variants and identified some types or locations of arteries or branching points with geometrical characteristics that are more likely to impair the hemodynamic balance within the circle of Willis. Some variants have a higher potential to comprise a cerebrovascular risk factor than others; for example, hypoplastic arteries presented higher resistance to flow and more imbalanced bifurcation geometry whereas accessory vessels presented less modified geometric characteristics. In line with our findings, David and Moore reported a prevalence of $40 \%$ for hypoplastic arteries in brains with aneurysms as opposed to $22 \%$ in normal brains, whereas the prevalence of accessory vessels in brains with aneurysms was practically similar to that of normal brains (18\% and $17 \%$, respectively) (11). Zaninovich et al. found a correlation between an incomplete circle of Willis and the incidence of ischemic or hemorrhagic stroke (41).

The presence of incisures in our model radar charts indicates an effect of the high resistance to flow on the vascular conductance throughout the circle of Willis. Those incisures represent the arterial segments with the poorest relative conductance in the arterial circle. In our specimens, the incisures correspond to the posterior communicating artery in the normal variant and to the hypoplastic arterial segments in the anatomical variations, such as the posterior and anterior cerebral arteries. Studies have shown that variants of the circle of Willis that include hypoplastic arteries affect cerebral perfusion in carotid stenosis (5), and during aortic surgery $(27,28)$. According to Pappantchev et al., in cases of hypoplastic PCA, the territory of the homolateral MCA and PCA is at risk of hypoperfusion, and in patients with hypoplastic ACA, the territory at risk is the one supplied by the homolateral ACA and the contralateral ACA and MCA (33). In cases of ICA occlusion, hypoplastic PCoA is linked to an increased risk of stroke (22).

Another example is the association between the presence of hypoplastic, asymmetric, or atypical arteries and the departure from branching optimality. These types of arteries tend to increase the risk of formation and rupture of aneurysms at their branching points $(13,25,26)$. A suboptimal branching of the bifurcations is linked to a higher wall shear stress (20). Prolonged exposure to wall shear stress induces adaptive responses in the endothelial cells, which favor the atherogenesis and degeneration of the arterial wall (29). This can also cause a higher rate of aneurysmal bleeding in patients with anatomical variations (26,31). Asymmetrical A1 and PCoA have been reported to be associated to $A C O A$ and $P C O A$ aneurysms, respectively (31). Moreover, we found that the bifurcations presenting the most imbalanced geometry, such as the carotid bifurcation, the A1-A2 junction, or the PCA$\mathrm{PCoA}$ junction, correspond to the most frequent localization of aneurysms and atherosclerosis $(25,29)$. A previous study 
conducted on a series of aneurysm patients reported statistically significant correlations between the aneurysm rates and the presence of anatomical variations in the ACoA, PCoA, and MCA (31). Bourcier et al. found that aneurysm patients, with or without a family history of aneurysms, presented a significant difference in the angle between the A1 and A2 segments on the aneurysmal side compared to the contralateral side, which is not observed in healthy individuals (4).

To our knowledge, this is the first study on variants of the circle of Willis that integrates resistance, conductance, and bifurcation geometry. Previous studies reporting differences in either resistance to flow $(1,3)$ or branching geometry $(20,36)$, presented them according to the anatomical location within the arterial circle. The novelty of the present study consisted of observing how these parameters behaved among different variation types. We defined a new parameter-the percentage departure from branching optimality $(B)$-in order to better compare the branching geometry. Asymmetric vessels differ significantly from their homologues in length or diameter, but cannot be classified as hypoplastic, which explains why the resistance to flow is not highly affected in these variants. However, an unusual diameter or length influences the position of the flow divider in the branching points and, consequently, the branching optimality. Hypoplastic vessels have the highest resistance to flow among all the variants due to their small diameter, which also increases the departure from branching optimality. In arteries with atypical origin, the length and diameter are not affected; hence resistance to flow remains normal. The departure from branching optimality can be affected or not depending on how the variant influences the flow divider position in the branching points. This explains why the mean value for $B$ in the vessels with atypical origin is higher than the general mean value, but lower than the mean values for asymmetrical and hypoplastic vessels.

We acknowledge the fact that using formalin-fixed brains could have limited the study since the fixation process can potentially change the arteries' volume. This limitation was accounted for by using the same fixation condition for all the specimens and by using ratios instead of absolute values. The diameters of the arteries provided by our study are consistent with those of other cadaveric studies (22), but they are not comparable with those of imaging studies as we measured outer diameters whereas imaging studies measure the diameter of the blood column. Except for the ICA, the diameters in our study are almost double-sized compared to those reported in a previous MR angiography study (39). Since our study was conducted on cadaver brains without cerebrovascular pathology, a direct link between the modified geometry and the appearance of neurological pathology could not be demonstrated. However, we developed a risk assessment method that can be used in prospective studies with animal models $(34,35)$ or imaging data from human subjects, which may be useful to prove this connection. Nevertheless, recognizing the anatomical variant $(15,31)$ or building a brain perfusion and wall shear stress model according to our method-which is quite simple and does not require any expensive software-could indirectly guide the clinician toward potential cerebrovascular risks when facing a patient with a specific anatomical variant.

\section{- CONCLUSION}

The presence of anatomical variations in the circle of Willis can modify the hemodynamics of the cerebral blood flow. Specific anomalies influence cerebral hemodynamics differently. This study proposes a simple algorithm to assess the hemodynamic effects of particular anatomical variants and applies it on some examples of circle of Willis variations with different hemodynamic behaviors.

\section{ACKNOWLEDGEMENT}

This research was carried out with funding from "Iuliu Hatieganu" University of Medicine and Pharmacy, Department of Anatomy and Embryology, Cluj-Napoca, Romania. Internal grant no. 4995/17/08.03.2016.

\section{REFERENCES}

1. Alastruey J, Parker KH, Peiró J, Byrd SM, Sherwin SJ: Modelling the circle of Willis to assess the effects of anatomical variations and occlusions on cerebral flows. J Biomech 40: 1794-1805, 2007

2. Alfano JM, Kolega J, Natarajan SK, Xiang J, Paluch RA, Levy El, Siddiqui AH, Meng H: Intracranial aneurysms occur more frequently at bifurcation sites that typically experience higher hemodynamic stresses. Neurosurgery 73: 497-505, 2013

3. Ansari S, Dadmehr M, Eftekhar B, McConnell DJ, Ganji S, Azari H, Kamali-Ardakani S, Hoh BL, Mocco J: A simple technique for morphological measurement of cerebral arterial circle variations using public domain software (Osiris). Anat Cell Biol 44: 324-330, 2011

4. Bourcier R, Lenoble C, Guyomarch-Delasalle B, DaumasDuport B, Papagiannaki C, Redon R, Desal H: Is there an inherited anatomical conformation favoring aneurysmal formation of the anterior communicating artery? J Neurosurg 126: 1598-1605, 2017

5. Cassot F, Zagzoule M, Marc-Vergnes JP: Hemodynamic role of the circle of Willis in stenoses of internal carotid arteries. An analytical solution of a linear model. J Biomech 33: 395-405, 2000

6. Cebral JR, Raschi M: Suggested connections between risk factors of intracranial aneurysms: A review. Ann Biomed Eng 41:1366-1383, 2013

7. Cecchi E, Giglioli C, Valente S, Lazzeri C, Gensini GF, Abbate R, Mannini L: Role of hemodynamic shear stress in cardiovascular disease. Atherosclerosis 214: 249-256, 2011

8. Cieslicki K, Ciesla D: Investigations of flow and pressure distributions in physical model of the circle of Willis. J Biomech 38: 2302-2310, 2005

9. Cucchiara B, Detre J: Migraine and circle of Willis anomalies. Med Hypotheses 70: 860-865, 2008

10. Cucchiara B, Wolf RL, Nagae L, Zhang Q, Kasner S, Datta R, Aguirre GK, Detre JA: Migraine with aura is associated with an incomplete circle of Willis: Results of a prospective observational study. PLoS One 26: e71007, 2013

11. David T, Moore S: Modeling perfusion in the cerebral vasculature. Med Eng Phys 30: 1227-1245, 2008 
12. Devault K, Gremaud PA, Novak V, Olufsen MS, Vernieres G, Zhao P: Blood flow in the circle of Willis: Modeling and calibration. Multiscale Model Simul 27: 888-909, 2008

13. Dimitriu CrP, Ionescu C, Bordei P, Bulbuc I: The role of anatomical anomalies in anterior communicating artery aneurysm rupture. ARS Med Tomit 3: 147-153, 2013

14. Grinberg L, Anor T, Cheever E, Madsen JR, Karniadakis GE: Simulation of the human intracranial arterial tree. Philos Trans A Math Phys Eng Sci 13: 2371-2386, 2009

15. Gunnal SA, Farooqui MS, Wabale RN: Anatomical variations of the circulus arteriosus in cadaveric human brains. Neurol Res Int 2014: 687281, 2014

16. Hall JE: Guyton and Hall Textbook of Medical Physiology, 12th ed. Elsevier, Saunders, 2010

17. Hartkamp MJ, van Der Grond J, van Everdingen KJ, Hillen B, Mali WP: Circle of Willis collateral flow investigated by magnetic resonance angiography. Stroke 30: 2671-2678, 1999

18. Hendrikse J, van Raamt AF, van der Graaf $Y$, Mali WP, van der Grond J: Distribution of cerebral blood flow in the circle of Willis. Radiology 235: 184-189, 2005

19. Hoksbergen AW, Majoie CB, Hulsmans FJ, Legemate DA: Assessment of the collateral function of the circle of Willis: Three-dimensional time-of-flight MR angiography compared with transcranial color-coded duplex sonography. Am J Neuroradiol 24:456-462, 2003

20. Ingebrigtsen T, Morgan MK, Faulder K, Ingebrigtsen L, Sparr $\mathrm{T}$, Schirmer $\mathrm{H}$ : Bifurcation geometry and the presence of cerebral artery aneurysms. J Neurosurg 101: 108-113, 2004

21. Iqbal S: A comprehensive study of the anatomical variations of the circle of Willis in adult human brains. J Clin Diagn Res 7: 2423-2427, 2013

22. Jalali Kondori B, Azemati F, Dadseresht S: Magnetic resonance angiographic study of anatomic variations of circle of Willis in a population in Tehran. Arch Iran Med 20: 235-239, 2017

23. Kamath S: Observations on the length and diameter of vessels forming the circle of Willis. J Anat 133: 419-423, 1981

24. Karatas A, Yilmaz H, Coban G, Koker M, Uz A: The anatomy of circulus arteriosus cerebri (circle of Willis): A study in Turkish population. Turk Neurosurg 26: 54-61, 2016

25. Kayembe KN, Sasahara M, Hazama F: Cerebral aneurysms and variations in the circle of Willis. Stroke 15: 846-850, 1984

26. Lazzaro MA, Ouyang B, Chen M: The role of circle of Willis anomalies in cerebral aneurysm rupture. J Neurointerv Surg 4: 22-26, 2012

27. Liang F, Fukasaku K, Liu H, Takagi S: A computational model study of the influence of the anatomy of the circle of Willis on cerebral hyperperfusion following carotid artery surgery. Biomed Eng Online 10:84, 2011
28. Lodi CA, Ursino M: Hemodynamic effect of cerebral vasospasm in humans: A modeling study. Ann Biomed Eng 27: 257-273, 1999

29. Nixon AM, Gunel M, Sumpio BE: The critical role of hemodynamics in the development of cerebral vascular disease. J Neurosurg 112: 1240-1253, 2010

30. Nordon, DG, Rodrigues Júnior OF: Variations in the brain circulation - the circle of Willis. J Morphol Sci 29: 243-247, 2012

31. Orakdogen M, Emon ST, Somay H, Engin T, Is M, Hakan T: Vascular variations associated with intracranial aneurysms. Turk Neurosurg 27: 853-862, 2017

32. Papantchev V, Hristov S, Todorova D, Naydenov E, Paloff A, Nikolov D, Tschirkov A, Ovtscharoff W: Some variations of the circle of Willis, important for cerebral protection in aortic surgery-a study in Eastern Europeans. Eur $\mathrm{J}$ Cardiothorac Surg 31: 982-989, 2007

33. Papantchev V, Stoinova V, Aleksandrov A, TodorovaPapantcheva D, Hristov S, Petkov D, Nachev G, Ovtscharoff $\mathrm{W}$ : The role of Willis circle variations during unilateral selective cerebral perfusion: A study of 500 circles. Eur J Cardiothorac Surg 44: 743-753, 2013

34. Pascalau R, Aldea CC, Padurean VA, Szabo B: Comparative study of the major white matter tracts anatomy in equine, feline and canine brains by use of the fibre dissection technique. Anat Histol Embryol 45: 373-385, 2016

35. Pascalau R, Szabo B: Fibre dissection and sectional study of the major porcine cerebral white matter tracts. Anat Histol Embryol 46: 378-390, 2017

36. Rossitti S, Löfgren J: Optimality principles and flow orderliness at the branching points of cerebral arteries. Stroke 24:10291032, 1993

37. Szabo B, Szabo I, Crişan D, Stefănuţ C: Idiopathic orbital inflammatory pseudotumor: Case report and review of the literature. Rom J Morphol Embryol 523: 927-930, 2011

38. Szabo BA, Pascalau R, Padurean VA: Morphometric study of the human brainstem and its neurovascular relations. Turk Neurosurg 2017 (Epub Ahead of print)

39. Yeniceri IO, Cullu N, Deveer M, Yeniceri EN: Circle of Willis variations and artery diameter measurements in the Turkish population. Folia Morphol (Warsz) 76: 420-425, 2017

40. Zamir M: Local geometry of arterial branching. Bull Math Biol 44: 597-607, 1982

41. Zaninovich OA, Ramey WL, Walter CM, Dumont TM: Completion of the circle of Willis varies by gender, age, and indication for computed tomography angiography. World Neurosurg 106: 953-963, 2017 